\title{
Mechanisms of irreversible decoherence in solids
}

\author{
F. D. Domínguez, R. C. Zamar, H. H. Segnorile, and C. E. González \\ FaMAF, Universidad Nacional de Córdoba, Córdoba X5000HUA, Argentina \\ and IFEG, CONICET, Córdoba X5000HUA, Argentina \\ (Received 26 April 2017; published 19 June 2017)
}

\begin{abstract}
Refocalization sequences in nuclear magnetic resonance (NMR) can in principle reverse the coherent evolution under the secular dipolar Hamiltonian of a closed system. We use this experimental strategy to study the effect of irreversible decoherence on the signal amplitude attenuation in a single-crystal hydrated salt where the nuclear spin system consists of the set of hydration water proton spins having a strong coupling within each pair and a much weaker coupling with other pairs. We study the experimental response of attenuation times with temperature, crystal orientation with respect to the external magnetic field, and rf pulse amplitudes. We find that the observed attenuation of the refocalized signals can be explained by two independent mechanisms: (a) evolution under the nonsecular terms of the reversion Hamiltonian, and (b) an intrinsic mechanism having the attributes of irreversible decoherence induced by the coupling with a quantum environment. To characterize (a) we compare the experimental data with the numerical calculation of the refocalized NMR signal of an artificial, closed spin system. To describe (b) we use a model of the irreversible adiabatic decoherence of spin pairs coupled with a phonon bath which allows evaluating an upper bound for the decoherence times. This model accounts for both the observed dependence of the decoherence times on the eigenvalues of the spin-environment Hamiltonian, and the independence from the sample temperature. This result, then, supports the adiabatic decoherence induced by the dipole-phonon coupling as the explanation for the observed irreversible decay of reverted NMR signals in solids.
\end{abstract}

DOI: 10.1103/PhysRevB.95.224423

\section{INTRODUCTION}

A cutting-edge subject in the research of irreversible processes is the study of the quantum dynamics of manyparticle interacting systems coupled with a quantum-correlated environment. In this scenario quantum decoherence represents a fascinating problem with links to fundamentals, as well as to modern application fields. In the first area, decoherence is considered by many as the mechanism responsible for the emergence of the classical world from the microscopic quantum mechanical world $[1,2]$. In the other, implementation of applications such as scalable quantum registers demands handling collective coherent states. The coherence that can be prepared in a multiparticle cluster becomes fragile due to coupling with the environment, turning it crucial to getting insight on the subtle mechanism by which the coherence loss occurs. Such process, which involves no energy exchange with the outer world, is called adiabatic quantum decoherence [3].

Nuclear magnetic resonance (NMR) of spin ensembles in the solid state can serve as a suitable test bed for the quantum dynamics on large clusters of interacting particles. In fact, NMR provides a variety of techniques to create and manipulate coherent spin states. In particular, a class of refocusing (often called "reversion") experiments allows retrieving the multispin dynamics governed by the dipole-dipole interaction of an ideal closed spin system. Actual experiments, however, yield refocused signals whose amplitudes attenuate with the reversion time. The source of such attenuation can be connected to experimental causes, and, from a microscopic viewpoint, to the unavoidable coupling of the observed system with other degrees of freedom [4-6]. In this work we use reversion experiments to isolate these effects by monitoring the signal attenuation times as a function of controlled experimental variables: efficiency of the reversion pulse sequences, sample temperature, and orientation with respect to the external magnetic field.

The explanation of irreversible decoherence in solid state NMR, and the role played by the environment in this process, has remained as an open question for a long time [7]. In particular, the mechanism by which nuclear spins are able to achieve a state of quasiequilibrium continues to be elusive. The NMR literature seldom relates the signal attenuation in reversion experiments in solids with the environmentinduced destruction of the coherent superposition of states (environmental decoherence). It is generally argued that strongly interacting spin systems, such as solids, are well isolated from the environmental degrees of freedom (thermal fluctuations), which manifests as "very long" spin-lattice relaxation times. This generally leads to expecting negligible effect of the environment on the spin dynamics over the earlier time scale. However, this fact does not rule out the occurrence of quantum decoherence, which involves the loss of local phases, within a time regime where the spin-lattice energy exchange is still ineffective. On the contrary, in the field of open quantum systems, it is currently accepted that the many-body character of the observed systems is a decisive condition for the occurrence of system-environment correlation, associated with the entanglement and adiabatic quantum decoherence of the observed system [3,6,8-10]. Such mechanism has a characteristic time scale much shorter than that of thermalization or spin-lattice relaxation, and can be thought of as a main microscopic source of the signal decay in reversion experiments and also in the occurrence of quasiequilibrium (or pseudothermalization) states [5,6,11].

A recent theoretical proposal describes the irreversible adiabatic decoherence of a system of weakly interacting spin pairs coupled with a phonon field [11]. The model considers that all spins in the sample are part of a complex dipolar network 
while the system-environment interaction is the fluctuation in the strongest dipolar couplings due to the low-frequency phonons. The resulting time dependence of the reduced density matrix elements is a product of the corresponding element of an isolated spin system and a decoherence function which introduces an irreversible decay. The rate of this decay increases with the Hamming distance between the involved states and with the intrapair dipolar coupling intensity. This coherence loss can be reflected in the decay of the expectation values that represent the system observables, and consequently in the measured signal amplitude.

In this work we use the well-known "magic echo" NMR reversion sequence (ME) on a single-crystal sample of CaSO4.2H2O (dihydrated calcium sulphate or gypsum), as a good representation of the ideal system treated in [11]. Our goal is to expose the main causes that attenuate the echo signal. We analyze two mechanisms very different in nature: the experiment nonideality, predominantly given by the evolution under nonsecular terms of the reversion Hamiltonian, and the environment-induced adiabatic decoherence.

The decay time dependence on the intrapair dipolar frequency and temperature is contrasted with theoretical estimates. To quantify the role of the nonsecular terms we compute their effect on the signal amplitude attenuation on an artificial ten-spin closed system having the same symmetry and orientations as the measured sample. The contribution of decoherence is interpreted in terms of predictions from the theory of open quantum systems, using the variation of the system purity as a quantifier of decoherence [12]. On this basis we calculate the purity, using the reduced density matrix from the pair-phonon model [11] and a reasonable hypothesis for the multispin correlation growth in the dipolar network. The purity also is an upper bound for the observable NMR signal; then, comparison of the calculated purity rates with the experimental attenuation times allows us to conclude that the pair-phonon model provides a proper explanation of the signal loss in the solid state NMR reversion experiments and consequently of the development of quasiequilibrium states.

\section{THE SAMPLE}

Gypsum $\left(\mathrm{CaSO}_{4} \cdot 2 \mathrm{H}_{2} \mathrm{O}\right)$ is a paradigmatic example of a hydrated salt, whose crystal structure was resolved long ago and redetermined more recently [13]. The unit cell is monoclinic, with $a, b, c=6.28 \AA, 15.20 \AA, 6.52 \AA$, and $\beta=127.4^{\circ}$. The sample used in this study is a piece of natural, transparent gypsum single crystal, with the size $\left(A=10 \times B=2 \times C=12 \mathrm{~mm}^{3}\right)$, where $A, B$, and $C$ are parallel to the primitive cell axes $a, b$, and $c$, respectively. It was placed on a holder which allows rotating the crystal around the $c$ axis, perpendicular to the external field $\vec{B}_{0}$, as shown in Fig. 1. Let us call $\varphi$ the angle between axis $b$ and $\vec{B}_{0}$, and define $\varphi=0$ when both vectors are parallel.

Gypsum has only one observable spin species: the hydration water protons, which adopt two different orientations. The geometry of regularly distributed spin pairs entails a hierarchy of dipolar interactions which can roughly be grouped into stronger (mainly produced by the intrapair interactions) and weaker. The NMR spectrum of this arrangement shows a splitting $\omega_{D}$ (Pake doublet) which depends, to first order,

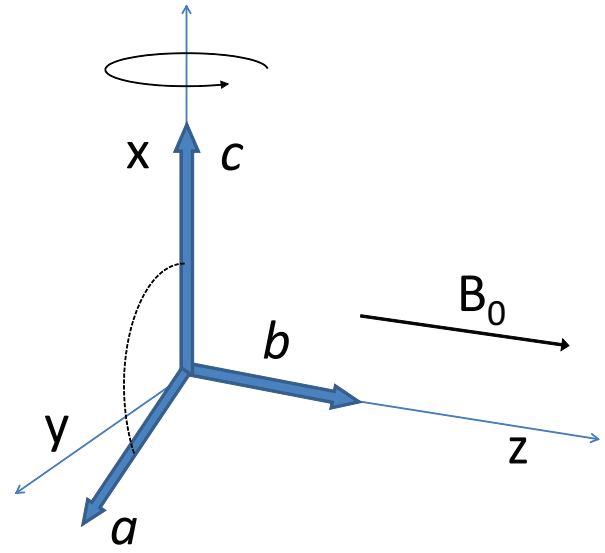

FIG. 1. Orientation of the single crystal with respect to the external magnetic field used in the experiment. The sample can be rotated around axis $c$.

on the magnitude of the dipolar intrapair interaction and consequently on the sample orientation with respect of the Zeeman field [14]. In our experiment we take advantage of this fact to manipulate $\omega_{D}$ by rotating the sample around a direction perpendicular to $\vec{B}_{0}$.

The spectra corresponding to the six different orientations, and all the experiments shown in this work, were measured on a Bruker minispec mq-20 spectrometer, at $20 \mathrm{MHz}$. The six spectra shown in Fig. 2 were fitted with Gaussian functions; the relation $\omega_{D}(\varphi)$ was determined from the dipolar splitting of the spectra and is shown in the second column of Table I. Spectra corresponding to angles $\varphi=0$ and 10 have two clearly resolved, asymmetric peaks, as expected in a system of weakly interacting spin pairs [15]. The tabulated frequencies correspond to the vertical lines at $\omega_{D}= \pm 23$ and $\pm 21 \mathrm{kHz}$ shown in Figs. 2(a) and 2(b), respectively. The spectrum of $\varphi=20^{\circ}$ has resolved symmetric peaks. The spectra of orientations $\varphi=30,35$, and 40 degrees are more complex because the water molecules are clearly not equivalent; the central peak seen in Figs. 2(d), 2(e), and 2(f) corresponds to one kind of molecules and the resolved doublets to the other. Again, we considered the largest frequency splitting to define $\omega_{D}$. The right column of Table I shows the second moments, $M_{2}$, of gypsum spectra obtained as the sum of intrapair $\left(\omega_{D}^{2} / 4 \pi^{2}\right)$ and interpair (width of each doublet component) contributions.

TABLE I. Experimental dipolar splitting $\omega_{D}$ for each crystal orientation $\varphi$ and the respective second moment $M_{2}$ of the NMR spectra of gypsum.

\begin{tabular}{lcc}
\hline \hline$\varphi(\mathrm{deg})$ & $\omega_{D} \pm 2(\mathrm{kHz} / 2 \pi)$ & $M_{2} \pm 5\left(\mathrm{kHz}^{2}\right)$ \\
\hline 0 & 46 & 1320 \\
10 & 42 & 1170 \\
20 & 36 & 990 \\
30 & 26 & 620 \\
35 & 18 & 315 \\
40 & 15 & 170 \\
\hline \hline
\end{tabular}



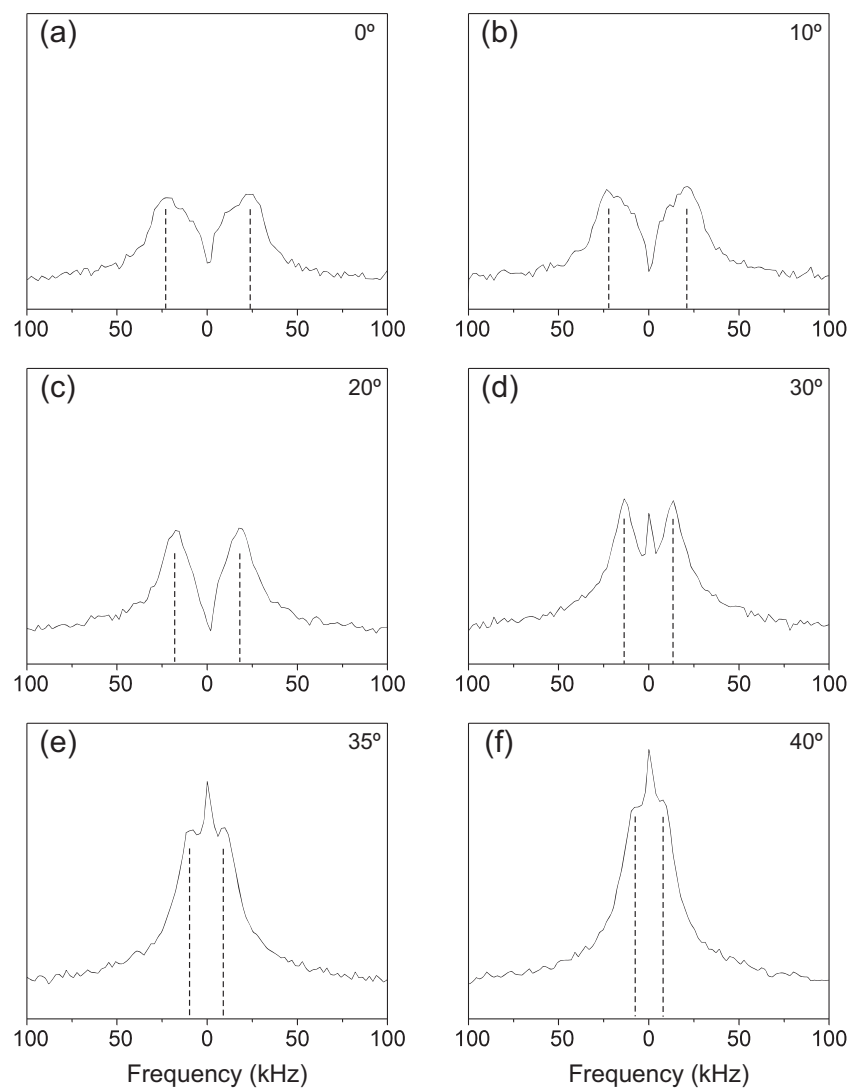

FIG. 2. Spectra of a gypsum single crystal with axis $c$ perpendicular to the magnetic field and axis at six orientations of axis $c$ with respect to the magnetic field. The spectra show resolved doublets for $\varphi=0,10,20$ degrees, while those corresponding to $\varphi=30,35,40$ degrees also have a central peak.

\section{MEASUREMENT OF DECOHERENCE}

\section{A. The magic echo sequence}

We use the well-known radio frequency pulse sequence called the "magic echo" (ME) [16], which has the effect of refocusing the time evolution due to the secular dipolar interaction of dipole-coupled spin systems in a large magnetic field. The ME sequence is shown in Fig. 3. The reversion block is composed of two pulses with amplitude $\omega_{1}$ (in frequency units), length $\alpha$, and alternating phases $x,-x$, which are "sandwiched" by two $\frac{\pi}{2}$ hard pulses of phases $y$ and $-y$. Alternating phases have the effect of avoiding accumulation of phase errors [16].

The effect of this block can be clearly shown by considering a simplified sequence with only one pulse with phase $x$,

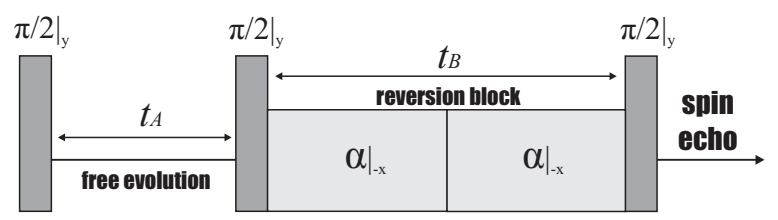

FIG. 3. Pulse sequence used in the experiment. The block of duration $t_{B}$ reverts the evolution under the secular dipolar Hamiltonian which takes place during $t_{A}$. duration $t_{B}$, and intensity $\omega_{1}$ inside the sandwich. The corresponding propagator is

$$
U_{M E}=R_{y}\left(\frac{\pi}{2}\right) \exp \left[-i t_{B}\left(\omega_{1} I_{x}+\mathcal{H}_{D}^{0}\right)\right] R_{y}\left(-\frac{\pi}{2}\right),
$$

where $R_{y}(\beta)=e^{-i \beta I_{y}}$ represent the hard pulses and $\mathcal{H}_{D}^{0}=$ $\sum_{k, j} \sqrt{1 / 6} \omega_{D}^{k j} T_{20}^{k j}$ is the total secular dipolar energy. $T_{20}^{k j}=$ $2 / \sqrt{6}\left(3 I_{z}^{(k)} I_{z}^{(j)}-\mathbf{I}^{(k)} \cdot \mathbf{I}^{(j)}\right)$ is the irreducible tensor operator corresponding to spins $k$ and $j$. Using the relations

$$
\begin{aligned}
A e^{B} A^{-1} & =e^{A B A^{-1}}, \\
e^{-i \frac{\pi}{2} I_{y}} I_{x} e^{i \frac{\pi}{2} I_{y}} & =-I_{z}, \\
e^{-i \frac{\pi}{2} I_{y}} T_{20}^{k, j} e^{i \frac{\pi}{2} I_{y}} & =-\frac{1}{2} T_{20}^{k, j}+\sqrt{\frac{3}{8}}\left(T_{22}^{k, j}+T_{2-2}^{k, j}\right),
\end{aligned}
$$

where $T_{2 \pm 2}^{k, j}=2 I_{ \pm}^{(k)} I_{ \pm}^{(j)}$, we have

$$
\begin{aligned}
U_{M E}= & \exp \left(-i \omega_{1} t_{B}\left\{-I_{z}+\sum_{k, j} \sqrt{\frac{1}{6} \frac{\omega_{D}^{k, j}}{\omega_{1}}}\right.\right. \\
& \left.\times\left[-\frac{1}{2} T_{20}^{k, j}+\sqrt{\frac{3}{8}}\left(T_{22}^{k, j}+T_{2-2}^{k, j}\right)\right]\right\} .
\end{aligned}
$$

If the $\mathrm{rf}$ intensity $\omega_{1}$ is bigger than the intrapair dipolar interaction $\omega_{D}$ (the main interaction in a spin pair system)

$$
\omega_{1} \gg \omega_{D} \geqslant \omega_{D}^{k, j},
$$

one can within first-order perturbation theory disregard the evolution caused by the nonsecular term $\left(T_{22}+T_{2-2}\right)[16,17]$. Since $\left[T_{20}, I_{z}\right]=0$ it is usual to set the experiment such that

$$
U_{M E}^{0} \simeq \exp \left[+i t_{B} \frac{1}{2} \mathcal{H}_{D}^{0}\right] .
$$

This expression brings about the main benefit of the ME, which is to revert the sign of the time evolution under the secular dipolar Hamiltonian and then "undo" one-half of its action. The typical experiment starts with the spin system in thermal equilibrium $\rho\left(0^{-}\right) \propto I_{z}$. The first, saturating $\left(\frac{\pi}{2}\right)$, rf pulse of phase $y$ at time $t=0$ leaves $\rho\left(0^{+}\right) \propto I_{x}$. The system evolves freely (under $\mathcal{H}_{D}^{0}$ ) during $t_{A}$ and backwards during $t_{B}$. Selecting $t_{B}=2 t_{A}$ will ideally revert the evolution to its state at $t=0$, and the NMR signal $\operatorname{Tr}\left\{\rho I_{x}\right\}$ should recover its initial amplitude.

The simple pulse sequence described above contains the essence of the reversion methods. Of course, there are various uncontrollable experimental settings that may overshadow the ideal response. There are many other ingenious sequences based on the ME which combine more ME modules with different phases that prevent or at least mitigate some of the various possible nonidealities of the actual experiment. It is clear, however, that the efficiency of the reversion sequence is restricted by condition (4), and that the experimental realization of this condition may not be possible for strong dipolar couplings. This may cause that part of the observed time evolution be due to the nonsecular term, which may enhance the degradation of the observed echoes. 


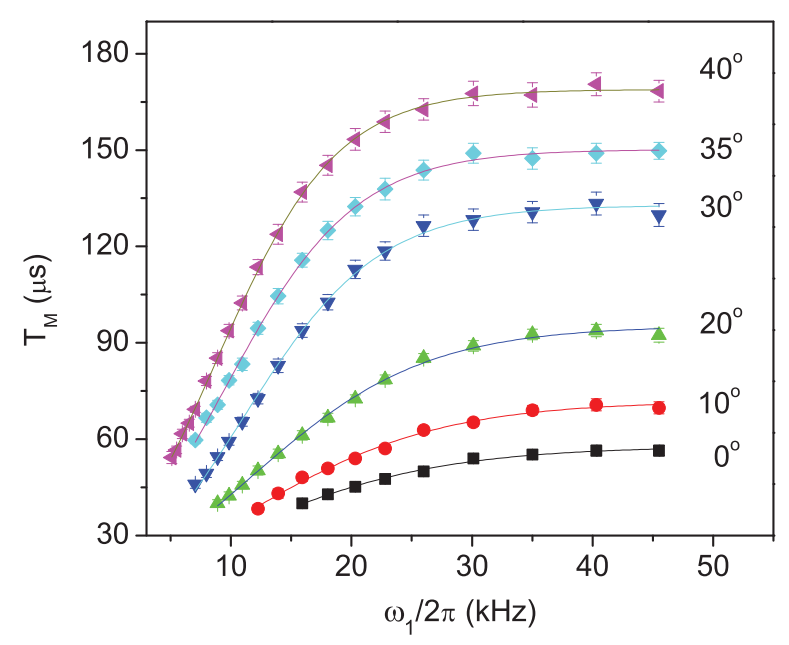

FIG. 4. Attenuation times $T_{M}$ of the reverted signal amplitudes as a function of the irradiation intensity $\omega_{1}$ of the ME sequence. Different curves (symbols) correspond to each studied orientation of the single-crystal sample with respect to the external magnetic field. Solid lines are fittings to a sigmoid $A\left(1+e^{-C\left(\omega_{1}+B\right)}\right)^{-1}$.

The purpose of this work is to isolate the different mechanisms that attenuate the reverted echo in a reversion experiment. Particularly, we look for a sign of the adiabatic decoherence due to the coupling of the spin system with the environment.

\section{B. Experimental results}

The signal amplitude recorded at the end of the ME sequence (see Fig. 3) attenuates as a function of the elapsed time $t_{A}$ (and consequently of $t_{B}$ also) with a characteristic time constant $T_{M}$ at which the signal amplitude reduces to $1 / e$ of its initial value. According to Eq. (3), one can expect the reversion sequence efficiency to depend on both the intrapair frequency $\omega_{D}$ (Table I) and the radiation intensity $\omega_{1}$; therefore we measured the attenuation time $T_{M}$ of the reverted signals for different values of $\omega_{1}$ and also varied $\omega_{D}$ by changing the sample orientation with respect to the external field.

Figure 4 shows the dependence of $T_{M}$ on $\omega_{1}$. Each curve corresponds to a fixed crystal orientation, then, to a given value of $\omega_{D}$. The experiment was performed at $T=310 \mathrm{~K}$. The fact that $T_{M}$ is very short for small $\omega_{1}$ at all crystal orientations is consistent with a poor reversion efficiency for low values of $\omega_{1} / \omega_{D}$, as follows from Eq. (3). All the data curves rise with an approximately linear trend whose slope depends on the sample orientation. The salient characteristic is that all the curves show a plateau at higher values of $\omega_{1}$, which implies that the reversion efficiency cannot be improved by increasing the alternating phase pulse amplitude in the ME sequence. The maximum values of $T_{M}$ attained by the different curves have a marked dependence on $\omega_{D}$. In fact, the data are adequately fitted (solid lines in Fig. 4) by sigmoids of the form

$$
T_{M}=\frac{A}{1+e^{-C\left(\omega_{1}+B\right)}}=\left(\frac{e^{-C B}}{A} \frac{1}{e^{C \omega_{1}}}+\frac{1}{A}\right)^{-1},
$$

which strongly suggests that the signal loss is dominated by two different processes: one that depends exponentially on

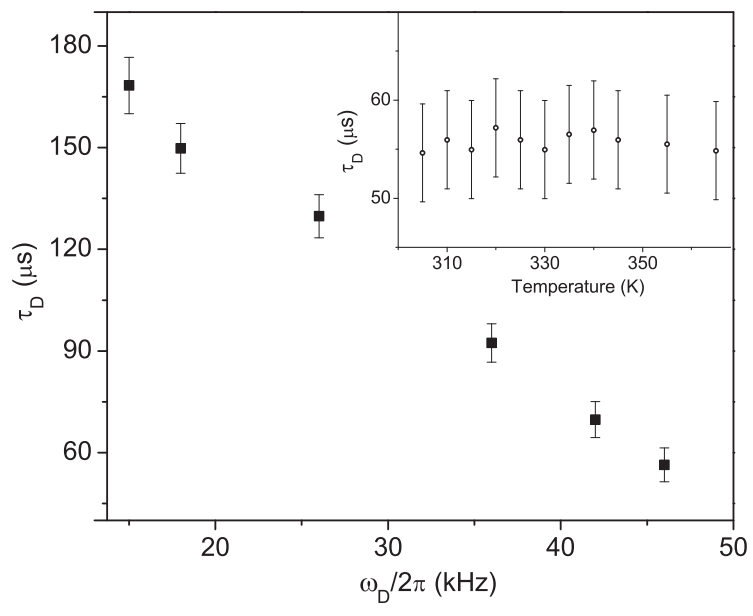

FIG. 5. Maximum attenuation times of reverted signals, $\tau_{D}$, vs the dipole frequency splitting of the NMR spectra corresponding to the six crystal orientations $\varphi=0,10,20,30,35,40$ degrees. Inset: Temperature dependence of $\tau_{D}$ at $\varphi=0$.

$\omega_{1}$, and another that is independent of $\omega_{1}$. We associate the former with evolution under the nonsecular terms of Eq. (3) that are not reverted with the ME sequence, and the latter with a different process, independent of the experimental settings.

Concerning the temperature dependence of the attenuation times, the inset of Fig. 5 shows $T_{M}$ measured at the maximum available $\omega_{1}$ and $\varphi=0^{\circ}$. They are noticeably temperature independent in a wide range, since the corresponding $T_{M} \mathrm{~s}$ are identical within the experimental error. It is worth mentioning that this behavior contrasts with the spin-lattice relaxation time whose value changes significantly in the same temperature range.

\section{Effect of the nonsecular terms}

Data from Fig. 4 measured in a wide range of dipolar couplings and radiation intensities allowed isolating the different sources of decay. Following the reasoning after Eq. (6), we assume that the two attenuation sources are independent and write the measured $T_{M}$,

$$
\frac{1}{T_{M}}=\frac{1}{T_{N S}}+\frac{1}{\tau_{D}},
$$

where $1 / T_{N S}$ is the decay rate due to the nonsecular part of the ME operator $U_{M E}$ in Eq. (3) and $1 / \tau_{D}$ is the decoherence rate. Other sources of signal decay, such as inhomogeneity of the rf field or finite width of the hard pulses, are optimized so they become negligible (besides, they are independent of $\omega_{1}$ and $\left.\omega_{D}\right)$.

In order to try this hypothesis we follow two steps. First, it is worth noticing that $\tau_{D}$, which represents the spin-environment coupling, does not depend on a purely experimental parameter as $\omega_{1}$, and also that the rate $1 / T_{N S} \rightarrow 0$ if $\omega_{1} \rightarrow \infty$. This allows us, in principle, to identify $\tau_{D}$ with the plateaus (maximum attenuation time) of the measured curves. Figure 5 shows that $\tau_{D}$ decreases for increasing values of $\omega_{D}$ in an approximately linear trend. We notice that $\tau_{D}$ may be slightly underrated for the angles $\varphi=0^{\circ}$ and $10^{\circ}$, because the quotient $\omega_{1} / \omega_{D}$ is low within the available rf intensity range. 


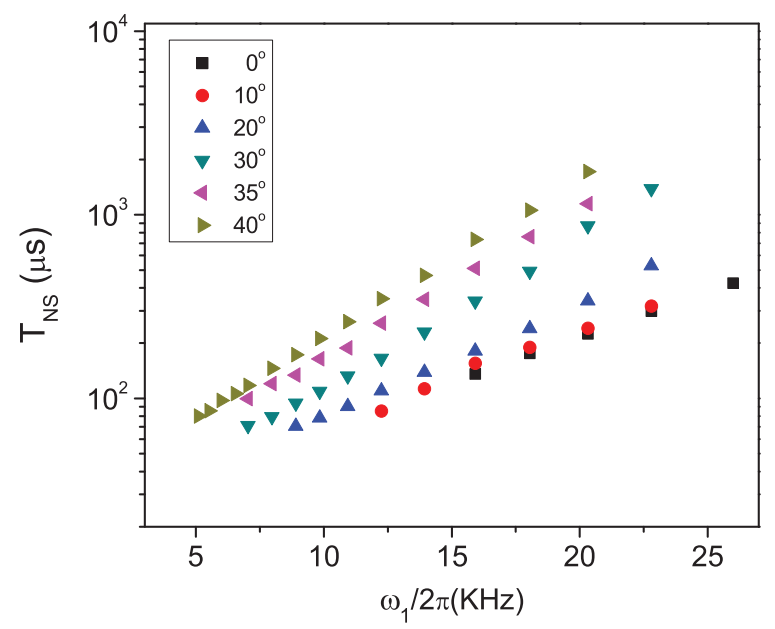

FIG. 6. Decay times $T_{N S}$ obtained from experimental data using Eq. (7) within the region where the dependence on $\omega_{1}$ is dominated by the nonreverted terms of the ME propagator.

Having determined $\tau_{D}$, we calculate the dependence of the decay time $T_{N S}$ on $\omega_{1}$. The results are shown in Fig. 6. The linearity of the plotted data indicates that $T_{N S}\left(\omega_{1}\right) \propto e^{\kappa \omega_{1}}$, with $\kappa$ an arbitrary value that depends on the crystal orientation $\varphi$. We excluded the higher values of $\omega_{1}$ because the functional form of Eq. (7) introduces significant errors within this frequency range.

As a second test, we analyze the validity of assigning $T_{N S}$ to the nonsecular terms of Eq. (3). Then, we compute their effect on the time evolution of the density matrix of a simulated sample of 10 spins located at the sites of the ${ }^{1} \mathrm{H}$ nuclei in a perfect lattice of $\mathrm{CaSO}_{4} \cdot 2 \mathrm{H}_{2} \mathrm{O}$ (it is worth mentioning that the size of this small cluster suffices to calculate the main frequency components the NMR spectra). As an example, the dipolar couplings for $\varphi=0^{\circ}$ are listed in Table II, and were calculated using the geometrical information from Ref. [13]. We simulate the experiment by calculating the density matrix

TABLE II. Dipolar couplings of the simulated sample of 10 spins, for the case $\varphi=0$.

\begin{tabular}{ccccccccc}
\hline \hline$k$ & $j$ & $\omega_{D}^{k j}\left(\frac{\mathrm{kHz}}{6 \pi}\right)$ & $k$ & $j$ & $\omega_{D}^{k j}\left(\frac{\mathrm{kHz}}{6 \pi}\right)$ & $k$ & $j$ & $\omega_{D}^{k j}\left(\frac{\mathrm{kHz}}{6 \pi}\right)$ \\
\hline 1 & 2 & 12.71 & 6 & 10 & -0.59 & 4 & 6 & 0.14 \\
3 & 4 & 12.71 & 7 & 10 & -0.49 & 3 & 8 & 0.14 \\
5 & 6 & 12.71 & 5 & 10 & -0.48 & 4 & 7 & 0.14 \\
7 & 8 & 12.71 & 6 & 9 & -0.48 & 1 & 8 & 0.12 \\
9 & 10 & 12.71 & 7 & 9 & 0.42 & 4 & 5 & 0.12 \\
8 & 10 & -2.34 & 4 & 9 & 0.41 & 1 & 10 & 0.11 \\
2 & 4 & -2.08 & 3 & 9 & 0.27 & 2 & 7 & 0.09 \\
6 & 8 & -2.08 & 2 & 9 & 0.25 & 3 & 6 & 0.09 \\
1 & 3 & -1.98 & 4 & 10 & 0.23 & 3 & 7 & 0.09 \\
5 & 7 & -1.98 & 4 & 8 & 0.22 & 1 & 7 & 0.08 \\
1 & 4 & -1.21 & 5 & 9 & 0.18 & 3 & 5 & 0.08 \\
2 & 3 & -1.21 & 3 & 10 & 0.18 & 1 & 6 & 0.05 \\
5 & 8 & -1.21 & 1 & 9 & 0.17 & 2 & 5 & 0.05 \\
6 & 7 & -1.21 & 2 & 10 & 0.17 & 2 & 6 & 0.05 \\
8 & 9 & 1.01 & 2 & 8 & 0.14 & 1 & 5 & 0.05 \\
\hline \hline
\end{tabular}

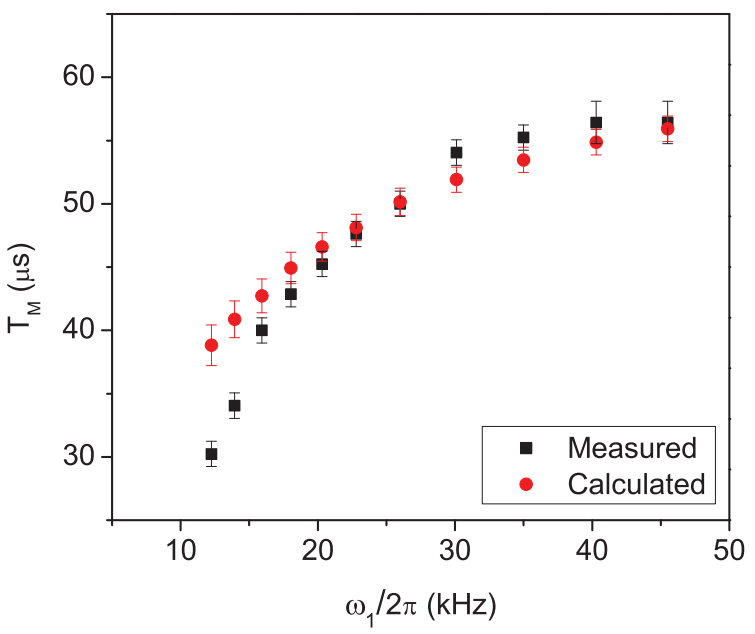

FIG. 7. Comparison between experimental and calculated values of $T_{M}=\left(T_{N \text { Scalc }}^{-1}+\tau_{D}^{-1}\right)^{-1}$, with $T_{N \text { Scalc }}$ calculated on a ten-spin sample, and setting $\tau_{D}=61 \mu \mathrm{s}$.

$\sigma(t)$ at time $t=t_{A}+t_{B}$ (see Fig. 3), and the corresponding reverted signal amplitude $\left\langle I_{x}\right\rangle=\operatorname{Tr}\left\{I_{x} \sigma(t)\right\}$, in a spin system which first evolves under the secular dipolar Hamiltonian, and then under the ME propagator of Eq. (3) according to the Liouville equation, for the different angles $\varphi$. In this way, we evaluate the efficiency of the reversion sequence, when the only source of attenuation is the evolution under the nonreverted terms of the dipolar Hamiltonian [nonsecular terms in Eq. (3)], which are weighted by $\omega_{1}$. Though the calculated signal exhibits a complex dependence on the total reversion time, its overall shape is an exponential decay with characteristic time $T_{N \text { Scalc }}\left(\omega_{1}, \omega_{D}\right)$ which depends on the irradiation amplitude and the dipolar frequency.

It is in principle possible to reproduce the measured data $T_{M}\left(\omega_{1}\right)$ by adding to the calculated rate $1 / T_{N \text { Scalc }}$ an extra contribution from decoherence. Figure 7 shows that the data calculated for $\varphi=0^{\circ}$ are remarkably similar to the experiment, even though the contribution $T_{N \text { Scalc }}$ was calculated in a small crystal of only ten spins. The slight discrepancy between $\tau_{D}=61 \mu$ s used in Fig. 7 and the experimental value $\tau_{D}=56 \mu$ s shown in Fig. 5 suggests either that the measured value may not correspond to the actual plateau, which could only be achieved by increasing the pulse intensity $\omega_{1}$, or that $T_{N \text { Scalc }}$ should be enhanced by computing the contribution of more spins. The small-size cluster may also explain the lower accuracy of the simulation, observed at $\varphi \neq 0$. However, the agreement found on the magnitudes and on the dependence with $\omega_{1}$ strongly supports hypothesis (7).

\section{Reverted signal amplitude in an open quantum system}

In this section we explore the relation between the experimental times $\tau_{D}$ and the irreversible adiabatic decoherence induced by coupling the observed system with an environment of harmonic phonons. In a recent work [11] we studied the adiabatic quantum decoherence in a system of interacting spins, coupled with a boson bath, in the framework of the theory of open quantum systems. The observed system is a network of weakly interacting spin pairs; the bath corresponds 
to lattice phonons, and the system-environment interaction is generated by the variation of dipole-dipole energy due to correlated shifts of the spin position, caused by phonons. The model includes secular dipolar interaction between all spins in the sample, but the arrangement in pairs naturally ranks them, since the intrapair interactions are larger than the interpair ones (provided that the pairs are sufficiently apart). Under the reasonable assumption that phonons are inefficient in producing transitions between spin levels, the time-evolution operator can be factorized as

$$
U(t)=V_{0}(t) V(t):=e^{-i t \mathcal{H}_{S}} e^{-i t\left(\mathcal{H}_{B}+\mathcal{H}_{I}\right)},
$$

where $\mathcal{H}_{S}$ is spin system energy, $\mathcal{H}_{B}$ is the phonon bath energy, and $\mathcal{H}_{I}$ is the dipolar energy variation due to spin-phonon coupling. The coherence loss is determined by the decoherence function $\Gamma(t)$,

$$
\begin{aligned}
\sigma_{m n}(t) & =\sigma_{0 m n}(t) e^{-\Gamma_{m n}(t)} \\
& =\left\langle m\left|V_{0}(t) \sigma(0) V_{0}^{\dagger}(t)\right| n\right\rangle \operatorname{Tr}_{B}\left\{V_{m}(t) \rho_{B} V_{n}^{\dagger}(t)\right\},
\end{aligned}
$$

where $|m\rangle$ and $|n\rangle$ are elements of an uncoupled-pairs spin basis $|m\rangle=\left|m_{1}, m_{2}, \ldots, m_{N}\right\rangle$, with $m_{k}$ the dipolar energy eigenvalue of the $k$ th pair. Additionally, it is assumed that only intrapair fluctuations of dipolar energy are relevant, since interpair interactions are weaker. In this way, the decoherence function takes the form

$$
\Gamma_{m n}(t)=\frac{a K_{B} T}{\mu c^{3}} M^{2} \omega_{D}^{2} t \approx 10^{-13} M^{2} \omega_{D}^{2} t,
$$

where $\omega_{D}$ is the intrapair dipolar interaction, $a$ is the distance between adjacent pairs, $K_{B}$ is the Boltzmann constant, $T$ is the absolute temperature, $\mu$ is the mass of the atom bearing the observed spin, $c$ is the speed of sound in the sample, and $M$ is the number of active pairs involved in the transition $|m\rangle=\left|m_{1}, \ldots, m_{N}\right\rangle \rightarrow|n\rangle=\left|n_{1}, \ldots, n_{N}\right\rangle$ (pair $j$ is active if $m_{j} \neq n_{j}$ ). By setting the values $a=1 \mathrm{~nm}, T=310 \mathrm{~K}$, $\mu=1.66 \times 10^{-27} \mathrm{~kg}$, and $c=3000 \mathrm{~m} / \mathrm{s}$, the decoherence function takes the form of the right term of Eq. (10). This is a simplified expression of the decoherence function, that considers phonons propagating only along the direction of the intrapair interaction, that is, those which perturb the dipolar coupling more efficiently. The equivalent $3 \mathrm{D}$ function has a more complex expression that also is a decreasing function with a similar decay rate.

In order to explore the implication of the decoherence model of Eq. (10) on the observed amplitudes of the reverted NMR signal, it would be necessary to calculate the expectation value $\left\langle I_{x}\right\rangle$ using the reduced density matrix of Eq. (9). Even though Eq. (10) provides the decay rate of each element, the calculation of all the terms involved in the observable signal is still not available. Besides, Eq. (10) is valid during free evolution, but there is not an analog under rf irradiation, that is, during the reversion block of the ME sequence. However, the theoretical analysis can still be carried out in terms of the system purity, $\mathcal{P}=\operatorname{Tr}\left(\sigma^{2}\right)$. This quantity is particularly useful because its variation can be interpreted as a quantifier of environment-induced decoherence [12], and can be estimated under reasonable hypotheses as shown below. At this point it is convenient to recall that the expectation value of any normalized operator $\langle\mathcal{O}\rangle$ is bounded for the square root of the purity because the trace is an inner product in the space of square complex matrices. Then, using the Cauchy-Schwarz inequality, we get

$$
\langle\mathcal{O}\rangle=\operatorname{Tr}(\sigma \mathcal{O}) \leqslant|\sigma||\mathcal{O}|=\sqrt{\operatorname{Tr}\left(\sigma^{2}\right)}=\sqrt{\mathcal{P}},
$$

provided $|\mathcal{O}|=\sqrt{\operatorname{Tr}\left(\mathcal{O}^{2}\right)}=1$. We can also safely assume that the purity of a spin state does not increase while the system is being irradiated because the rf pulses do not act on the lattice variables. So, we conclude that the purity just before the start of the reversion block is a good upper bound for the square of the maximum reverted signal. This reasoning enables us to study the dependence of phonon decoherence with the intrapair dipolar frequency $\omega_{D}$ and temperature $T$ through the behavior of the purity

$$
\mathcal{P}(t)=\sum_{m n}\left|\sigma_{0 m n}\right|^{2} e^{-2 \Gamma_{m n}(t)} .
$$

Each term of Eq. (12) involves a characteristic number of active pairs $M$, and $\Gamma$ depends on $m, n$ only through the value of $M$. So, we can rewrite $\mathcal{P}$ as

$$
\mathcal{P}(t)=\sum_{M} e^{-2 \Gamma_{M}(t)}\left(\sum_{\{m n\} \in \mathcal{M}}\left|\sigma_{0 m n}\right|^{2}\right),
$$

where the index $\{m n\} \in \mathcal{M}$ means that the sum involves states $\{m, n\}$ having $M$ active pairs.

The main obstacle in calculating $g(M):=\sum_{\{m n\} \in \mathcal{M}}\left|\sigma_{0 m n}\right|^{2}$ is the half knowledge of the closed, many-spin dynamics, encoded in $\sigma_{0}$. After the first pulse in Fig. 3, the state $\sigma_{0}=I_{x}$ is a statistical mixture of single-spin, single-quantum states. The subsequent evolution $V_{0}$ under the secular dipolar Hamiltonian does not increase the coherence order, while its flip-flop term is actually capable of increasing the number of active spins. Thus an estimation of the number of active spins is needed, but a method for a direct calculation of this number is not available.

Inspired by the works by Cho et al. [18], and Levy and Gleason [19] (see Appendix), we represent $g(M)$ by a Gaussian distribution

$$
g(M)=\frac{1}{\Delta M} e^{-\frac{\left[M-M_{0}(t)\right]^{2}}{2 \Delta M^{2}}},
$$

whose center increases exponentially with time as

$$
M_{0}(t)=e^{R \sqrt{M_{2}} t},
$$

with $R$ an arbitrary constant and $M_{2}$ the second moment of the crystal. The width of the distribution grows with $M_{0}$ with a factor of proportionality $q$,

$$
\Delta M=q M_{0}(t) .
$$

Then, in the continuum limit

$$
\begin{aligned}
\mathcal{P}(t) & \propto \int_{0}^{\infty} e^{-\frac{2 K_{B} T a \omega_{D}^{2} M^{2} t}{m c^{3}}} g(M) d M \\
& \propto \int_{0}^{\infty} e^{-\frac{2 K_{B} T a \omega_{D}^{2} M^{2} t}{m c^{3}}} e^{-\frac{\left(M-M_{0}(t)\right)^{2}}{2 \Delta M^{2}}} \frac{d M}{\Delta M} .
\end{aligned}
$$

This expression gives a computable expression of the time dependence of the purity of a system of spin pairs coupled to a phonon bath. By performing a numerical integration, using 


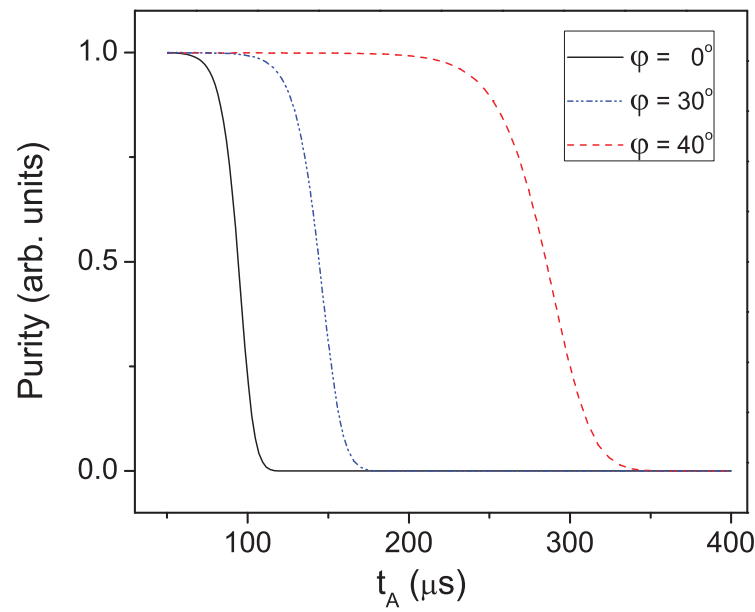

FIG. 8. Purity of the spin pairs system as a function of the free evolution time, at three different crystal orientation angles.

a trapezoidal rule, we find that $\mathcal{P}(t)$ is a decreasing sigmoid function that decays at different rates according to the crystal orientation as shown in Fig. 8. The different curves of purity correspond to the dipolar frequencies $\omega_{D}$ and second moments from Table I. We set $R=2$ and $\Delta M=0.2 M_{0}$ arbitrarily, to get insight into the dependence of $\mathcal{P}$ on $t$.

In order to give a qualitative description of the purity decay, let us characterize this decreasing function by the time $\tau_{P}$ at which the curve reaches the value $1 / e$. The dependence of $\tau_{P}$ on the arbitrary parameter $q$ from Eq. (16) is rather weak, as shown in Fig. 9(a) where the solid and dashed purity curves have similar characteristic times even when they correspond to very different values of $q$. Figure 9 (b) shows that $\tau_{P}$ has a different although moderate sensitivity to the parameter $R$ from Eq. (15) at different dipolar frequencies (angles).

Besides this, it is worth emphasizing that the calculated values of $\tau_{P}$ yielded by the theoretical prediction have the same order of magnitude as the experimental ones, even when Eq. (13) corresponds to a simplified one-dimensional model. Figure 10 shows that $\tau_{P}$ is in fact an upper bound for the measured $\tau_{D}$ (triangles), and that the purity decay time decreases with increasing dipolar frequency $\omega_{D}$ within the whole frequency range. The temperature dependence of $\tau_{P}\left(\omega_{D}\right)$ is depicted by the solid, dashed, and dotted curves in Fig. 10 which correspond to $T=220 \mathrm{~K}, T=310 \mathrm{~K}$, and $T=360 \mathrm{~K}$, respectively, and were calculated by setting $R=2$ and $q=0.2$. Their similarity indicates that $\tau_{P}$ is almost insensitive to temperature variation within this range. This fact is in complete agreement with the experimental results reported in Sec. III B. Since Eq. (13) remains valid at lower temperatures, one can estimate that temperatures lower than $40 \mathrm{~K}$ would be required in order to attain an observable (i.e., greater than a $10 \%$ experimental error) reduction of the acoustic phonon mechanism of irreversible decoherence.

It can be seen in Fig. 10 that the pair-boson model and the measured $\tau_{D}$ have a similar frequency dependence for $\omega_{D}>20 \mathrm{kHz}$. In the lower frequency instead, the model underestimates the mechanisms that determine the experimental decoherence times. It should be noticed that this low-frequency breakdown is consistent with the loss of the doublet shape in
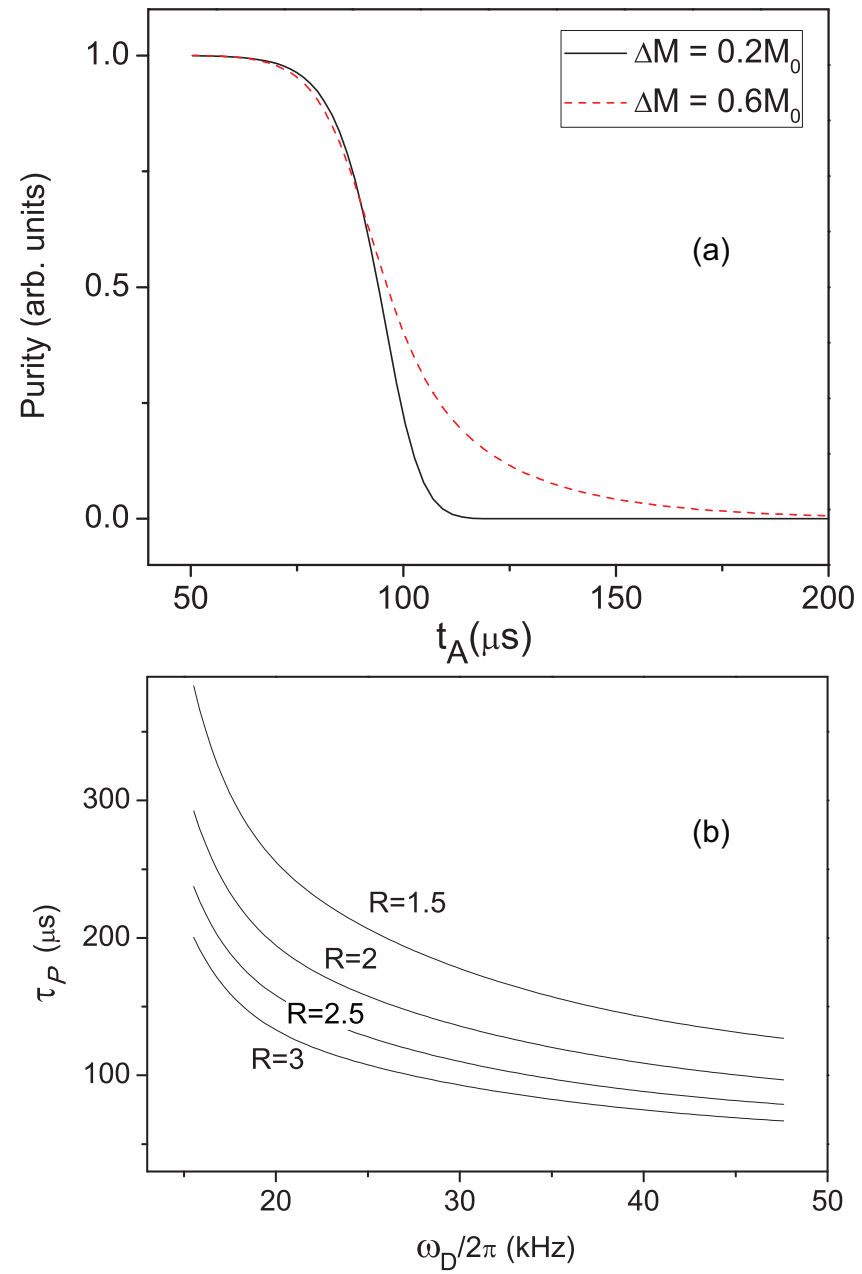

FIG. 9. (a) Time dependence of purity calculated from Eq. (17) with $q=0.2$ (solid) and $q=0.6$ (red dash). Both curves have very similar decay time. (b) Frequency dependence of the calculated decay time $\tau_{P}\left(\omega_{D}\right)$ for different values of $R$.

the NMR spectrum at the corresponding crystal orientations $\left(\varphi=35^{\circ}\right.$ and $\left.40^{\circ}\right)$. In other words, the pair-boson model may not describe the experiment because the spin system is actually not composed of easily distinguishable spin pairs. In summary, we interpret the results of Fig. 10 as an indication that the intrinsic mechanism which attenuates the amplitude of the reverted signals may be attributed to the adiabatic decoherence due to the coupling of a system of interacting pairs with a phonon bath. This subtle correlation, although inefficient as a mechanism of relaxation, can alternatively be the mechanism which drives adiabatic decoherence.

\section{CONCLUSIONS}

We studied the proton NMR signal amplitude response of a crystalline solid in reversion experiments. We find that the signal decay can be explained in terms of two main independent sources, one associated with the evolution under the nonsecular terms of the spin interactions that cannot be reverted in the experiment. This limitation can be mitigated to a high degree by performing the experiment under high-field conditions in the rotating frame. The other source of signal 


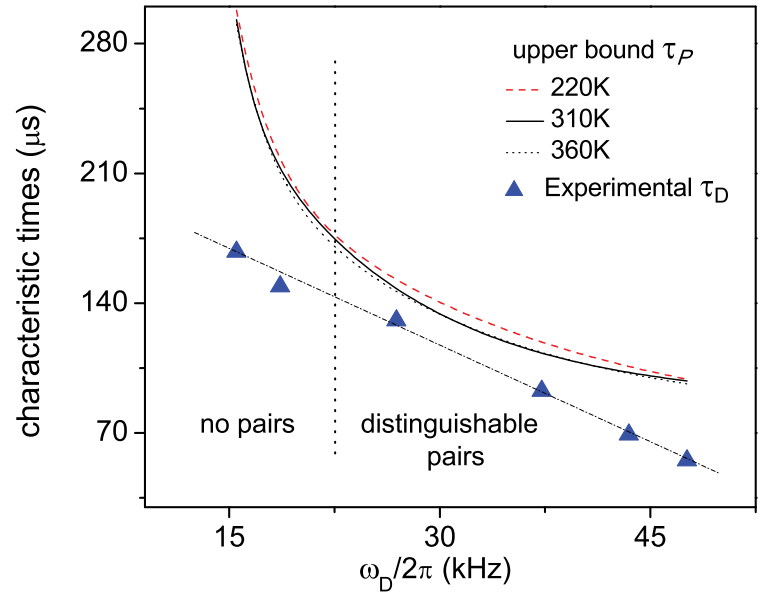

FIG. 10. Experimental decoherence times (blue triangles) measured at different values of the intrapair dipolar coupling. The solid (black) and dashed (red) curves are the higher bound for the decoherence times calculated with the pair-boson model for decoherence. The model as well as the experiment are insensitive to a change of $140 \mathrm{~K}$ in the sample temperature. The vertical line divides the regions where the NMR spectrum indicates the presence (or not) of well-separated intrapair and interpair dipolar interactions.

reduction has a fundamental character, and was ascribed to environment-induced decoherence.

We measured the characteristic attenuation times of the reverted signals under different experimental conditions. The data are well described by two contributions, one $\left(T_{N S}\right)$ that depends on both the intensities of the rf pulses in the reversion sequence, $\omega_{1}$ and $\omega_{D}$, and one $\left(\tau_{D}\right)$ which depends only on $\omega_{D}$. The salient aspects are that $\tau_{D}$ is (a) markedly sensitive to changes in the intrapair dipolar frequency, and (b) independent of the sample temperature within the probed range $310-360 \mathrm{~K}$. This behavior led us to propose that the irreversible decay $\tau_{D}$ originates in the adiabatic loss of quantum coherence mediated by the coupling of the dipole energy with low-frequency phonons.

With the aim of probing the microscopic mechanism underlying the signal decay, we calculate the reduced density matrix elements within the theory of open quantum systems. We estimated the spin system purity (which is an upper bound for the reverted signal amplitude) using the pair-phonon interaction model in its 1D version [11]. The model was supplemented with a statistical hypothesis to introduce the growth of the spin cluster. The resulting purity decay time $\tau_{P}\left(\omega_{D}\right)$ showed a strong dependence on the dipolar frequency. Its frequency dependence is similar to that of the signal within a range of crystal orientation angles where the sample can safely be regarded as a linear chain of weakly interacting pairs; in contrast, it deviates from the experimental trend for orientation angles where the separation into stronger and weaker dipolar couplings no longer applies. Within the region of discrepancy, the theoretical model underestimates the effectiveness of decoherence, revealing that the used model does not contemplate all the relevant mechanisms. Anyway, this simple model of pairs allowed us to show up a frequency dependence which is a signature of the quantum adiabatic decoherence. Likewise, the calculated purity is practically temperature independent. These features are in total consistency with the experimental characteristic times, which confirms that the observed irreversible decay can be explained by the adiabatic decoherence induced by the dipole-phonon coupling.

It is worth remarking that our results involve quantitative estimations of the effect of decoherence in solid state NMR, starting from first principles and using a model Hamiltonian that represents many properties of a real sample. This kind of study can facilitate new interesting applications related to vibrational properties of solids, growth of correlation in spin clusters, or protection of complex states against environmentinduced degradation.

\section{ACKNOWLEDGMENT}

This work was supported by Secretaría de Ciencia y Técnica, Universidad Nacional de Córdoba, H.H.S. and F.D.D. thank CONICET for financial support.

\section{APPENDIX}

Cho et al. [18] studied the growth of coherence orders on the $x$ basis, in a crystalline spin system. The spin state is described by the density operator $\sigma$, and evolves under $V_{0}(t)=e^{-i \mathcal{H}_{s} t}$, as in Eq. (9). Rotation about the $y$ axis (that is, changing to the $x$ basis) leaves the spin state as

$$
R_{y} \sigma(t) R_{y}^{-1}=R_{y} V_{0}(t) R_{y}^{-1} R_{y} \sigma(t=0) R_{y}^{-1} R_{y} V_{0}(t) R_{y}^{-1} .
$$

The evolution of the rotated state involves exciting higher coherences, because the rotated evolution operator $R_{y} V_{0}(t) R_{y}^{-1}$ is similar to a coherence pumping sequence. This growth is explained in the work by Levy and Gleason [19], where they showed that, under pumping sequences, the most populated coherence order raises exponentially with time, with a rate that depends on the second moment of the crystal [as Eq. (15)].

This view led us to assume that the growth of the active spin pairs under the influence of secular dipolar evolution in the state $\sigma(t)=V_{0}(t) \sigma(t=0) V_{0}(t)$ is similar to the growth of coherence order in a rotated state $R_{y} \sigma(t) R_{y}^{-1}$.
[1] E. Joos, Decoherence and the Appearance of a Classical World in Quantum Theory (Springer-Verlag, Berlin, Heidelberg, New York, 2003).

[2] W. H. Zurek, Rev. Mod. Phys. 75, 715 (2003).

[3] T. Yu and J. H. Eberly, Phys. Rev. B 66, 193306 (2002).

[4] C. Petitjean and P. Jacquod, Phys. Rev. Lett. 97, 124103 (2006).
[5] C. E. González, H. H. Segnorile, and R. C. Zamar, Phys. Rev. E 83, 011705 (2011).

[6] H. H. Segnorile and R. C. Zamar, J. Chem. Phys. 135, 244509 (2011).

[7] S. W. Morgan, V. Oganesyan, and G. S. Boutis, Phys. Rev. B 86, 214410 (2012). 
[8] D. Mozyrski and V. Privman, J. Stat. Phys. 91, 787 (1988).

[9] A. C. S. Costa, M. W. Beims, and W. T. Strunz, Phys. Rev. A 93, 052316 (2016).

[10] P. Haikka, T. H. Johnson, and S. Maniscalco, Phys. Rev. A 87, 010103(R) (2013).

[11] F. D. Domínguez, C. E. González, H. H. Segnorile, and R. C. Zamar, Phys. Rev. A 93, 022120 (2016).

[12] W. H. Zurek, S. Habib, and J. P. Paz, Phys. Rev. Lett. 70, 1187 (1993).

[13] J. C. A. Boeyens and V. V. H. Ichharam, Z. Kristallogr. - New Cryst. Struct. 217, 9 (2002).

[14] G. E. Pake, J. Chem. Phys. 16, 327 (1948).
[15] A. Keller, in Advances in Magnetic Resonance, Advances in Magnetic and Optical Resonance, Vol. 12, edited by J. S. Waugh (Academic Press, New York, 1988), pp. 183-246.

[16] W.-K. Rhim, A. Pines, and J. S. Waugh, Phys. Rev. B 3, 684 (1971).

[17] A. Abragam and M. Goldman, Nuclear Magnetism: Order and Disorder, International Series of Monographs on Physics (Clarendon Press, 1982).

[18] H. Cho, T. D. Ladd, J. Baugh, D. G. Cory, and C. Ramanathan, Phys. Rev. B 72, 054427 (2005).

[19] D. H. Levy and K. K. Gleason, J. Phys. Chem. 96, 8125 (1992). 